\title{
Impact of gain compression factor on modulation characteristics of InGaAs/GaAs self-assembled quantum dot lasers
}

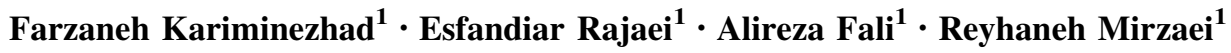

Received: 12 April 2016/ Accepted: 6 June 2016/Published online: 20 June 2016

(c) The Author(s) 2016. This article is published with open access at Springerlink.com

\begin{abstract}
This paper investigates the influence of gain compression factor on the modulation response of InGaAs/GaAs self-assembled quantum dot laser based on rate equations. For different gain compression factors the output power-current characteristics, light emissions of quantum dot laser have been simulated and effect of gain compression factor changes on quantum dot laser is illustrated. Also, small and large-signal response of quantum dot lasers is studied and the impact of the gain compression factor is presented. It explains that increase of gain compression factor, decreases small-signal modulation characteristics, nevertheless, improves large-signal response of quantum dot lasers. It helps to generate better laser signal quality, higher eye and smaller jitter. The large-signal behavior of a laser diode determines its capability for digital data transfer. The modulation speed of quantum dot lasers is of specific importance if such lasers are considered for optical communication systems.
\end{abstract}

Keywords Gain compression factor · Small signal modulation · Quantum dot lasers

\section{Introduction}

Several unique properties of quantum dot lasers make them attractive for many future optical communication applications. Quantum dots (QDs) have been proposed as a possible route to increase the modulation speed of semiconductor lasers. Since its first demonstration, self-assembled quantum

Esfandiar Rajaei

raf404@guilan.ac.ir

1 Department of Physics, Faculty of Science, University of Guilan, Rasht, Guilan, Iran dot lasers attracted great research interest due to their unique physical properties and quantum dot lasers have demonstrated several advantages for use in fiber optic communication systems. A QD laser has the potential to achieve very high differential gain, and the gain spectral width, to be reduced to less than that of planar quantum wells [1]. In contrast, because the planar quantum well has a continuous density of states, the room temperature, probability is small for its electrons and holes to occupy only those states that couple to the lasing mode. In principle, a QD active region can couple every injected electron and hole to the lasing mode so that the change in gain per change in injected electron is increased over a planar well [2]. The advantages of quantum dots over quantum wells are due to their unique density of states resulting from three-dimensional confinement of carriers [3]. Ultralow threshold current and high temperature stability has been demonstrated for $1.3 \mu \mathrm{m}$ selfassembled quantum dot (QD) lasers by many research groups [4-7]. It is also proved that QD lasers would have a large modulation bandwidth due to the high differential gain of three dimensional confined QD states [8].

The small-signal bandwidth of semiconductor lasers is commonly used to predict their large-signal modulation capabilities. The $1.3 \mu \mathrm{m}$ emission self-assembled InGaAs/ GaAs dots were first formed in 1994 by the alternate supply of Group-III and IV source materials with the quite low growth rate in metal-organic vapor phase epitaxy [9]. Some properties of self assembled quantum dot lasers (SAQDL) have been studied in [9-11]. Simulation of quantum dot lasers with two lasing states for InGaAs/GaAs quantum dot lasers emitting in $1.3 \mu \mathrm{m}$ wavelength is also presented in [12-14]. Similar studies for (113)B InAs/InP quantum dot lasers are considered in $[15,16]$ Note that at high bias condition, it has been shown in the aforementioned studies that the transition of lasing state occurs from the ground 
state to the excited state. Differential gain and linewidth enhancement factor are studied in $[17,18]$. The influence of the P-doping and tunneling injection on the modulation response of quantum dot lasers are studied in [2, 19]. Impacts of gain compression factor on dynamic and static characteristics quantum dot laser are presented in [20-23].

In the present work, a numerical method based on rate equations is used for modeling the InGaAs/GaAs SAQDL to analyze the light-current and modulation response of the QD-laser is presented. We present a thorough analysis of the modulation characteristics of InGaAs/GaAs SAQDL considering the gain compression factor, carrier relaxation and carrier escape. To our knowledge, this paper is the first one which describes the impact of gain compression factor on modulation response by solving the rate equations. Moreover, in this paper, we study the effect of gain compression factor in both small-signal and large-signal modulation response of $\mathrm{InGaAs} / \mathrm{GaAS}$ quantum dot lasers which have great importance in optical communication. It is shown that the increase of the gain compression factor causes reduction of modulation bandwidth and improves large-signal properties of quantum dot lasers.

In this paper, first the physics and theory of the InGaAs/ GaAs SAQDL is presented and then results are discussed. The role of gain compression factor is highlighted and it is shown that it plays an important role in the quantum dot laser modulation. Finally, conclusions are presented in the last section.

\section{Physics and modeling}

In the following, a numerical model is used to study carrier dynamics in the lowest energy level of an InGaAs/GaAs quantum dot system. In this model, dominant dynamics of carriers and photons such as carrier relaxation, capture and reexcitation rates to dots, radiative and nonradiative recombination rates of carriers, inhomogeneous broadening of dot resonant energy primarily due to the size fluctuation of dots are considered. The most popular and useful way to deal with the carrier and photon dynamics in lasers is to solve the rate equations for carriers and photons. Here, we present a coupled set of rate equations to calculate the lasing properties of quantum dot lasers. Our model describing the carrier dynamics in QD lasers is based on the experimental works [10, 24, 25]. It is assumed that only a single, discrete electron-hole ground state is formed inside a quantum dot and that charge neutrality always holds on each dot. Figure 1 shows the physical structure and scheme of the carrier transition process in the active region of self-assembled quantum dot lasers. In this figure, $N_{\mathrm{s}}$ and $N_{\mathrm{q}}$ are carrier numbers in separate confinement heterostructure (SCH) and wetting layer, respectively. We also consider that $N_{n}$ is the carrier number in the quantum dot. The

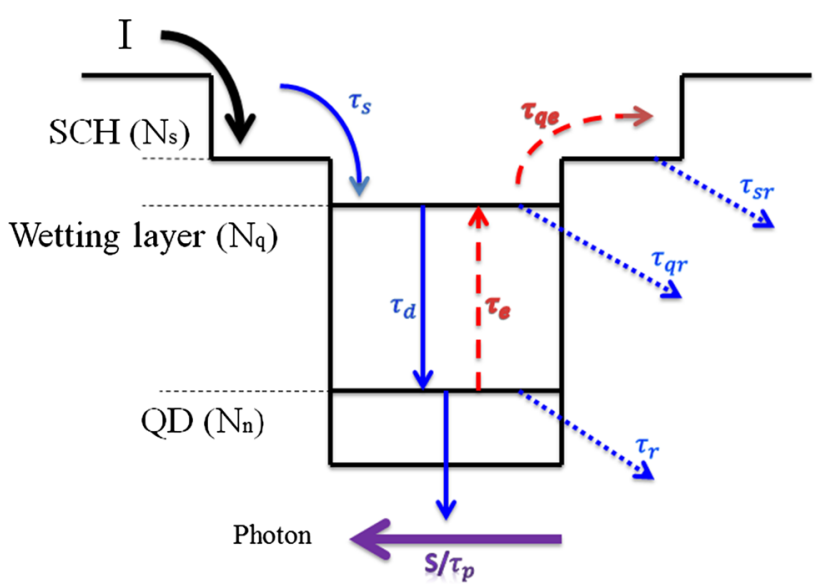

Fig. 1 Energy band diagram for the active region of $\mathrm{InGaAs} / \mathrm{GaAs}$ SAQDL

injected carriers diffuse through the $\mathrm{SCH}$ layer, relax into the quantum well $(\mathrm{QW})$, and then relax into the dot.

Above lasing threshold current, carriers in the ground state emit photons into the lasing mode primarily due to the stimulated emission process. To study the gain compression factor impact on quantum dot laser, for simplicity in this model, we neglect cavity internal modes and we consider a single mode rate equation model. The rate equations for the carrier-photon system are:

$\frac{\mathrm{d} N_{\mathrm{s}}}{\mathrm{d} t}=\frac{I}{e}-\frac{N_{\mathrm{s}}}{\tau_{\mathrm{s}}}-\frac{N_{\mathrm{s}}}{\tau_{\mathrm{sr}}}+\frac{N_{\mathrm{q}}}{\tau_{\mathrm{qe}}}$

$\frac{\mathrm{d} N_{\mathrm{q}}}{\mathrm{d} t}=\frac{N_{\mathrm{s}}}{\tau_{\mathrm{s}}}+\frac{N_{n}}{\tau_{\mathrm{e}}}-\frac{N_{\mathrm{q}}}{\tau_{\mathrm{qr}}}-\frac{N_{\mathrm{q}}}{\tau_{\mathrm{qe}}}-\frac{N_{\mathrm{q}}}{\tau_{\mathrm{d}}}$,

$\frac{\mathrm{d} N_{n}}{\mathrm{~d} t}=\frac{N_{\mathrm{q}}}{\tau_{\mathrm{d}}}-\frac{N_{n}}{\tau_{\mathrm{r}}}-\frac{N_{n}}{\tau_{\mathrm{e}}}-\frac{\left(c / n_{\mathrm{r}}\right) g_{\mathrm{tot}} \Gamma}{1+\varepsilon_{\mathrm{m}} \Gamma S / V_{\mathrm{a}}} S$,

$\frac{\mathrm{d} S_{\mathrm{m}}}{\mathrm{d} t}=\frac{\left(c / n_{\mathrm{r}}\right) g_{\mathrm{tot}} \Gamma}{1+\varepsilon_{\mathrm{m}} \Gamma S / V_{\mathrm{a}}} S-\frac{S}{\tau_{\mathrm{p}}}+\frac{\beta N_{n}}{\tau_{\mathrm{r}}}$.

Here, $V_{\mathrm{a}}$ is the active region volume $\beta$ is the spontaneous-emission coupling coefficient and $I$ is the injected current. The associated time constants are: diffusion time in the $\mathrm{SCH}$ region $\left(\tau_{\mathrm{s}}\right)$, carrier non-radiative recombination lifetime in the $\mathrm{SCH}$ region $\left(\tau_{\mathrm{sr}}\right)$, carrier re-excitation time from the quantum dot to the wetting layer $\left(\tau_{\mathrm{e}}\right)$, carrier nonradiative recombination lifetime in the wetting layer $\left(\tau_{\mathrm{qr}}\right)$, carrier relaxation lifetime into quantum dot $\left(\tau_{\mathrm{d}}\right)$ and nonradiative recombination lifetime in the quantum $\operatorname{dot}\left(\tau_{\mathrm{r}}\right)$. For simplicity, carrier re-excitation lifetime from the quantum well to the $\mathrm{SCH}$ region $\left(\tau_{\mathrm{qe}}\right)$ is neglected. The maximum optical gain at the center of the broadening function is given as [20]:

$g_{\mathrm{m}}^{(1)}=\frac{2 \pi e^{2} \hbar N_{\mathrm{D}}}{c n_{\mathrm{r}} \varepsilon_{0} m_{0}^{2}} \frac{\left|P_{\mathrm{cv}}^{\sigma}\right|^{2}}{E_{\mathrm{cv}} \Gamma_{\text {in hom }}}(2 P-1)$, 
where $\varepsilon_{0}=\Gamma_{\text {inhom }} / 2.35$ and the full-width at half maximum (FWHM) is $\Gamma_{\text {inhom }},\left|P_{\mathrm{cv}}^{\sigma}\right|^{2}$ is the transition matrix element, $n_{\mathrm{r}}$ is the refractive index, $E_{\mathrm{cv}}$ is the inter-band transition energy. According to Pauli's exclusion principle, occupation probability in the quantum dot ground state $P$ is related to $N$ as [22]:

$P=N /\left(2 N_{\mathrm{D}} V_{\mathrm{a}}\right)$,

where $N$ is the population of carriers in the dot and $N_{\mathrm{D}}$ is the dot density.

The maximum optical gain, including nonlinear susceptibility, is written as [26]:

$g_{\text {tot }}=g_{\mathrm{m}}^{(1)}+g_{\mathrm{m}}^{(3)}=g_{\mathrm{m}}^{(1)}\left[1-\varepsilon_{\mathrm{m}} \Gamma S / V_{\mathrm{a}}\right] \cong \frac{g_{\mathrm{m}}^{(1)}}{1+\varepsilon_{\mathrm{m}} \Gamma S / V_{\mathrm{a}}}$,

where $\varepsilon_{\mathrm{m}}$ is the third-order nonlinear coefficient [27].

$\varepsilon_{\mathrm{m}}=\frac{h q^{2}}{2 \pi n_{r}^{2} m_{0}^{2} \varepsilon_{0}} \frac{\left|P_{\mathrm{cv}}^{2}\right|}{\hbar \omega} \frac{\tau_{\mathrm{p}}}{F_{\mathrm{ho}}}$

Coverage factor is considered as:

$\xi=N_{\mathrm{D}} V_{\mathrm{D}}$,

where $V_{\mathrm{D}}$ is the volume of a dot. The photon lifetime in the cavity $\tau_{\mathrm{p}}$ is given as:

$\tau_{\mathrm{p}}^{-1}=\left(c / n_{\mathrm{r}}\right)\left[\alpha_{\mathrm{i}}+\ln \left(1 / R_{1} R_{2}\right) /\left(2 L_{\mathrm{ca}}\right)\right]$,

where $R_{1}$ and $R_{2}$ are the cavity mirror reflectivity coefficients and $\alpha_{\mathrm{i}}$ is the internal loss.
To calculate the small-signal modulation response of the laser we have applied the current $I=I_{0}+I\left(\omega_{\mathrm{m}}\right) \exp \left(i \omega_{\mathrm{m}} t\right)$ with a modulation angular frequency of $\omega_{\mathrm{m}}$, to the laser. By use of calculation of [21], the resultant modulation response function of the laser is given as:

$M\left(\omega_{\mathrm{m}}\right)=\frac{1}{\left(1+\omega_{\mathrm{m}}^{2} \tau_{\mathrm{d}}^{2}\right)^{1 / 2}\left(1+\omega_{\mathrm{m}}^{2} \tau_{\mathrm{s}}^{2}\right)^{1 / 2}} \frac{Q}{\left[\left(\omega_{\mathrm{m}}^{2}-\omega_{\mathrm{r}}^{2}\right)^{2}+\gamma^{2} \omega_{\mathrm{m}}^{2}\right]^{1 / 2}}$,

$\omega_{\mathrm{r}}^{2}=\frac{\Gamma S_{0} g^{\prime}}{\tau_{\mathrm{p}}\left(1+\varepsilon_{\mathrm{m}} \Gamma S_{0} / V_{\mathrm{a}}\right)}\left[1+\frac{\varepsilon_{\mathrm{m}} / V_{\mathrm{a}}}{g^{\prime} \tau_{\mathrm{r}}}\right]$,

$\gamma=\frac{\Gamma S_{0} g^{\prime}}{1+\varepsilon_{\mathrm{m}} \Gamma S_{0} / V_{\mathrm{a}}}+\frac{\varepsilon_{\mathrm{m}} \Gamma S_{0} / V_{\mathrm{a}}}{\tau_{\mathrm{p}}\left(1+\varepsilon_{\mathrm{m}} \Gamma S_{0} / V_{\mathrm{a}}\right)}+\frac{1}{\tau_{\mathrm{r}}}$,

$Q=\frac{\Gamma S_{0} g^{\prime}}{1+\varepsilon_{\mathrm{m}} \Gamma S_{0} / V_{\mathrm{a}}}$,

and $g^{\prime}$ is differential gain:

$g^{\prime}=\frac{c}{n_{\mathrm{r}}} \frac{\partial g_{\mathrm{m}}^{(1)}}{\partial N}$,

where $\omega_{\mathrm{r}}$ is the relaxation oscillation frequency and $\gamma$ is damping factor. The $3-\mathrm{dB}$ bandwidth limited by the relaxation lifetime of $\tau_{\mathrm{d}}$ is given as:

$f_{3 \mathrm{~dB}}=\left(2 \pi \tau_{\mathrm{d}}\right)^{-1}$

We have considered typical SAQDL material and geometrical parameters such as those given in Table 1 $[8,24,28]$.
Table 1 Parameters used in SAQDL modeling [8, 24, 28]

\begin{tabular}{lll}
\hline Symbol & Description & Value \\
\hline$\tau_{0}$ & Carrier relaxation lifetime & $10 \mathrm{ps}$ \\
$\tau_{\mathrm{s}}$ & Carrier capture time in SCH region & $1 \mathrm{ps}$ \\
$\tau_{\mathrm{sr}}$ & Spontaneous emission lifetime in SCH & $2.8 \mathrm{~ns}$ \\
$\tau_{\mathrm{qr}}$ & Carrier recombination lifetime in QW & $3 \mathrm{~ns}$ \\
$\tau_{\mathrm{r}}$ & Recombination lifetime in QD & $2.8 \mathrm{~ns}$ \\
$\tau_{\mathrm{qe}}$ & Carrier scape time from QD to WL & $3 \mathrm{~ns}$ \\
$R$ & Radius of a QD (Cylindrical shape) & $8 \mathrm{~nm}$ \\
$H$ & Height of a QD (Cylindrical shape) & $5 \mathrm{~nm}$ \\
$R_{1}$ & Right facet reflectivity & $30 \%$ \\
$R_{2}$ & Left facet reflectivity & $90 \%$ \\
$L_{\mathrm{ca}}$ & Cavity length & $900 \mu \mathrm{m}$ \\
$\Gamma$ & Optical confinement factor & $6 \%$ \\
$\alpha_{\mathrm{i}}$ & Intrinsic absorption coefficient & $6 \mathrm{~cm}^{-1}$ \\
$n_{\mathrm{r}}$ & Refractive index & 3.5 \\
$\beta$ & Spontaneous emission coupling coefficient & $10^{-4}$ \\
$\Delta$ & Spin-orbit interaction energy of QD material & $0.35 \mathrm{eV}$ \\
$E_{\mathrm{g}}$ & Band gap energy & $0.8 \mathrm{eV}$ \\
$V_{\mathrm{a}}$ & Active region volume & $2.2 \times 10^{-16} \mathrm{~m}^{3}$ \\
\hline & &
\end{tabular}




\section{Results and discussions}

By using the rate Eqs. (1)-(4) and Eq. (11), it is possible to study the dynamic characteristics and modulation response of InGaAs/GaAs quantum dot lasers. Results of simulations are presented in this section. Value of gain compression factor is chosen around $\varepsilon_{\mathrm{m}}=10^{-22} \mathrm{~m}^{3}$, which it corresponds to the regular formalism $[29,30]$.

In Fig. 2 photon density versus time for different gain comparison factors has been illustrated. As it can be seen, by increasing the gain compression factor, the carrier relaxation oscillation frequency and photon number has been reduced. It is related to the decrease of the optical gain of the quantum dot laser.

In Fig. 3 output power versus injected current for different gain compression factors have been presented. As we can see from Fig. 3, increase in the gain compression factor leads to decrease in output power and slope efficiency. It is due to the fact that when gain compression factor raises the nonlinear saturation gain reduces and it leads to decrease of differential gain that, it contributes to degrade in the output power and slope efficiency. Increase of gain compression factor does not affect the threshold current of quantum dot laser. It is because gain compression factor changes gain of the laser in the injection currents of the upper threshold current.

Gain compression factor, causes output power rollover in quantum dot lasers. In Fig. 3, increase of gain compression factor, reduces threshold of the output power rollover to lower injection currents and it starts in lower injection currents. For gain compression factor of $10 \times$ $10^{-22}$ and $20 \times 10^{-22}$ output power rollover appears at injection currents of around 7 and $3 \mathrm{~mA}$ respectively. Figure 4 shows the modulation response for different injected currents. It illustrates that, increase of injected

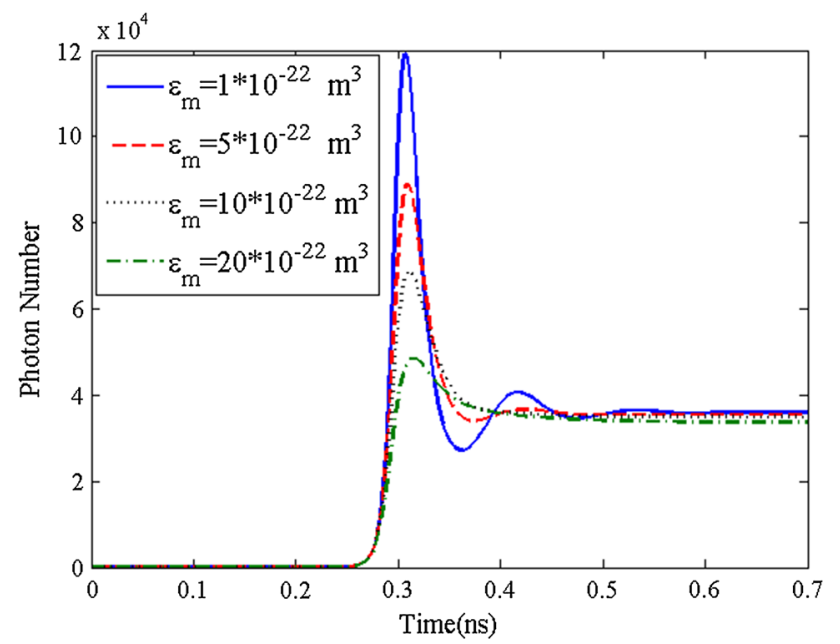

Fig. 2 Photon density versus time for various gain compression factors

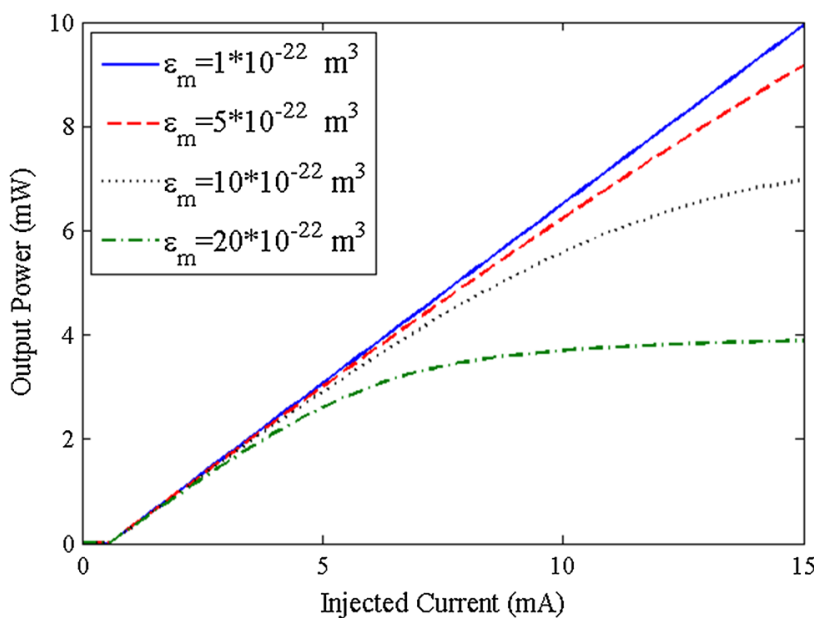

Fig. 3 Light current characteristics of quantum dot lasers for various values of gain compression factors

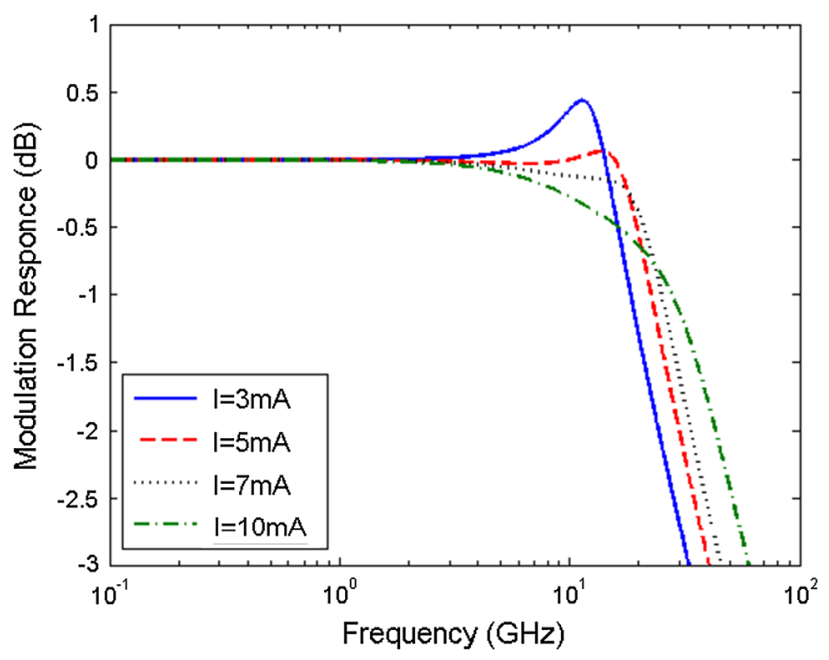

Fig. 4 Modulation response of quantum dot laser for different injected currents

current leads to increase modulation bandwidth and decrease relaxation oscillation frequency and peak amplitude of quantum dot laser. This corresponds to results of InAs/InP quantum dot lasers [31].

Quantum dot lasers suffer from larger gain compression factors $\left(10^{-22}-10^{-21} \mathrm{~m}^{3}\right)$ as compared to their quantum well counterparts $\left(10^{-25}-10^{-23} \mathrm{~m}^{3}\right)$ which can also alter the modulation dynamics [32]. Figure 5 illustrates modulation response for different gain compression factors. This figure indicates that relaxation oscillation frequency, peak amplitude, and modulation bandwidth reduce with increasing of the gain compression factor. The larger gain compression clearly suppresses the resonance peak and reduces the modulation bandwidth. The resonance frequency for $1 \times 10^{-22}$ is $11.4 \mathrm{GHz}$ and by increase of gain compression factor, it is decreased. 


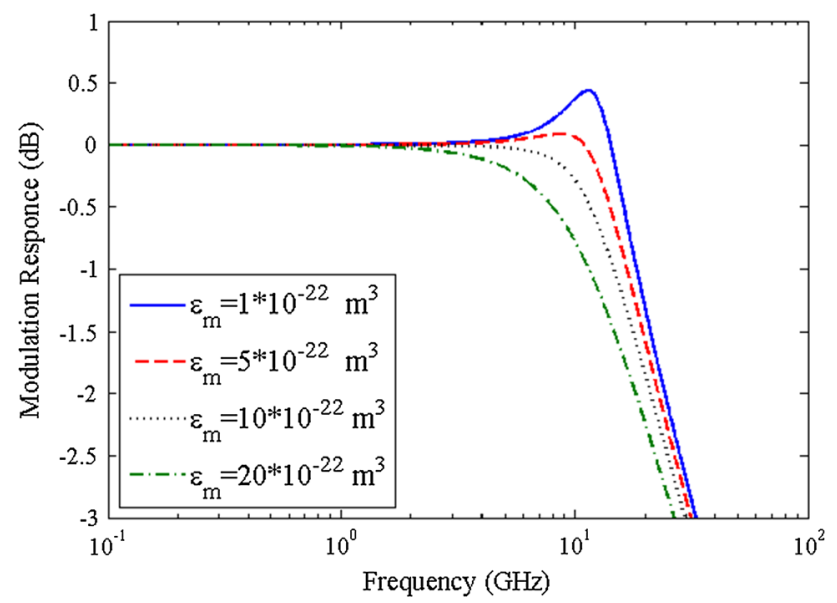

Fig. 5 Modulation response of quantum dot laser for different gain compression factors

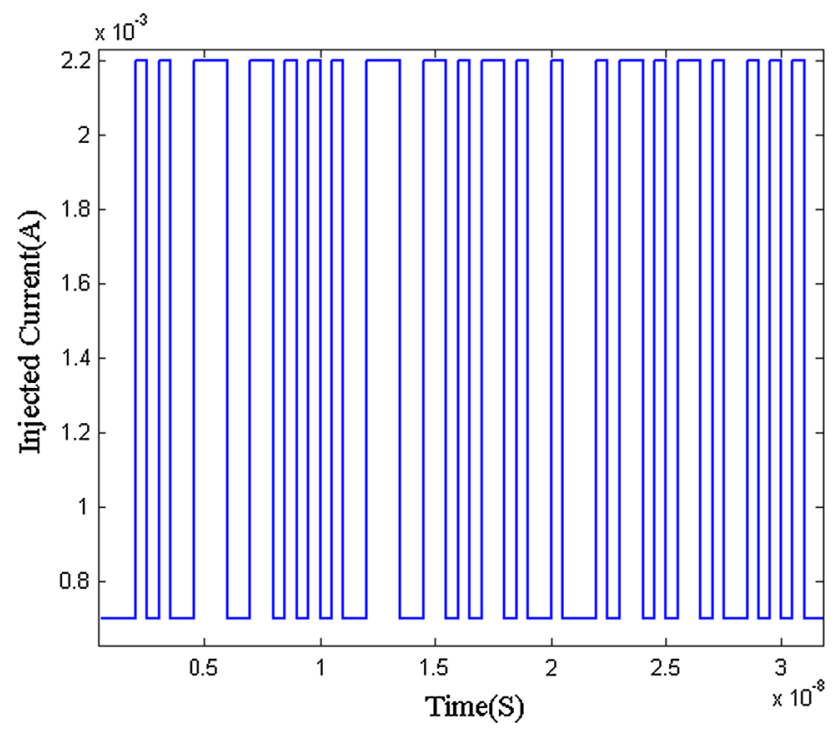

Fig. 6 Digital injected current versus time

To study large-signal properties of quantum dot lasers, we introduce a digital injected current to the laser. In Fig. 6, the injected current changes between 0.0007 and 0.0022 A every 0.5 ns. Figure 7 shows the digital photon number for the repetition frequency of $2 \mathrm{Gib} / \mathrm{s}$ in $1 \times$ $10^{-22} \mathrm{~m}^{3}$ gain compression factor.

In Fig. 7, it is demonstrated, when injected current increased to higher values of more than threshold current, after a small turn on delay, the photon number is increased to higher values.

By using digital injected current, it is possible to accurately model the measured eye diagram [33]. Figure 8a-c show eye diagram for gain compression factor with values

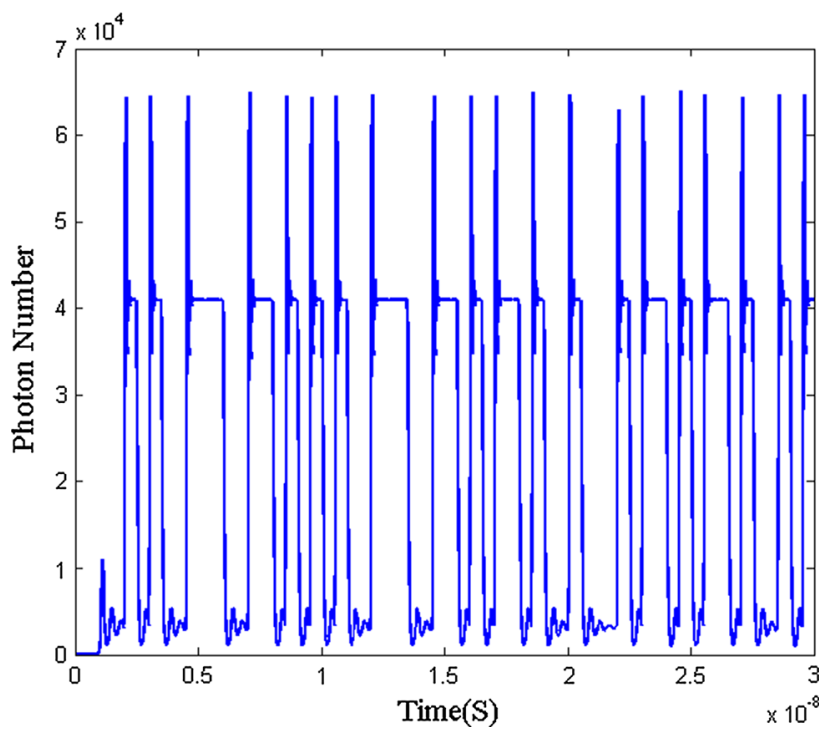

Fig. 7 Large-signal photon number of QD laser in $1 \times 10^{-22} \mathrm{~m}^{3}$ gain compression factor

of $1 \times 10^{-22}, 5 \times 10^{-22}$, and $10 \times 10^{-22} \mathrm{~m}^{3}$. It is shown that, for low gain compression factors the eye height is low. Increase of gain compression factor, appears the larger eye height and smaller jitter. The eye diagram is improved by increasing of gain compression factor. It is notable that the eye height is directly associated with the noise. By increasing gain compression factor, eye height increases and so eye becomes more open and noise decreases.

It is demonstrated that, quantum dot lasers with a large nonlinear gain compression factor have narrow small-signal bandwidth, but are capable of large-signal modulation at very high rates.

\section{Conclusions}

In this paper a theoretical model has been used to investigate the modulation properties of InGaAs/GaAs quantum dot laser. The numerical model takes into account gain compression factor. Variation of gain compression factor, does not affect ground state threshold current of the laser but it degrades the photon number and consequently, reduction of output power in the $\mathrm{InGaAs} / \mathrm{GaAs}$ quantum dot laser is demonstrated. It is shown that increase of the gain compression factor, decrease resonance frequency, relaxation oscillation frequency and modulation bandwidth. Despite gain compression factor limits small-signal response of the quantum dot lasers, the eye diagram of the large-signal modulation of the laser is improved for large values of gain compression factor. As we increase gain 
Fig. 8 Eye diagram of quantum dot laser diagram for the repetition frequency of $2 \mathrm{Gib} / \mathrm{s}$ in different gain compression factors
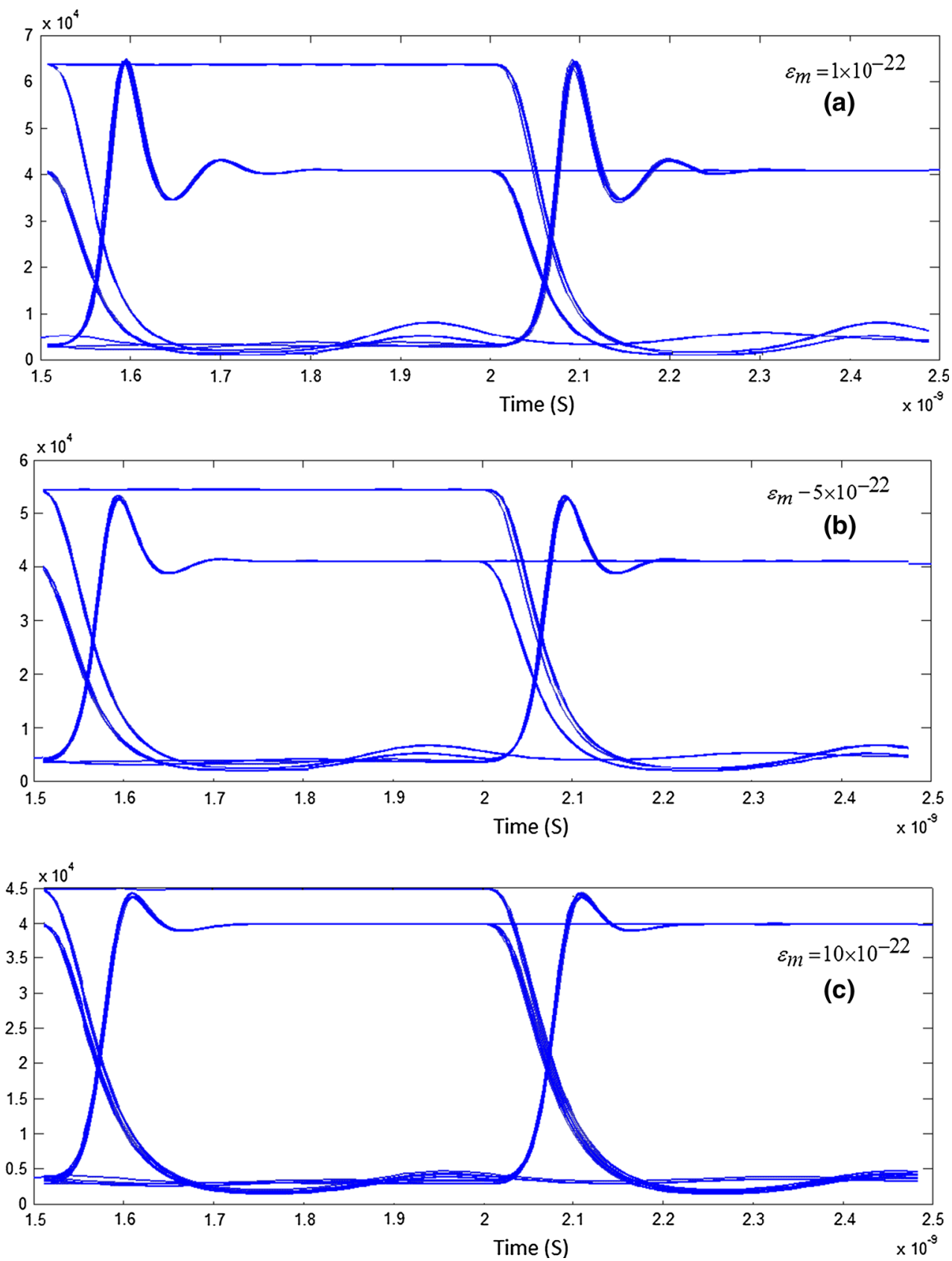

compression factor, we realize clear eye opening with small jitter. QD lasers with a high nonlinear gain compression factor have narrow small signal bandwidth, but are capable of large-signal modulation at very high rates.

Open Access This article is distributed under the terms of the Creative Commons Attribution 4.0 International License (http://crea tivecommons.org/licenses/by/4.0/), which permits unrestricted use, distribution, and reproduction in any medium, provided you give appropriate credit to the original author(s) and the source, provide a link to the Creative Commons license, and indicate if changes were made.

\section{References}

1. Vahala, K.: Quantum box fabrication tolerance and size limits in semiconductors and their effects on optical gain. IEEE J. Quantum Electron. 24, 523-530 (1988)

2. Deppe, D.G., Huang, H., Shchekin, O.B.: Modulation characteristics of quantum dot lasers: the influence of P-type doping and the electronic density of states on obtaining high speed. IEEE J. Quantum Electron. 38, 1587-1593 (2002)

3. Liu, H.Y., Childs, D.T., Badcock, T.J., Groom, K.M., Seller, I.R., Hopkinson, M., Hogg, R.A., Robbins, D.J., Mowbray, D.J., Skolnick, M.S.: High-performance three-layer $1.3 \mu \mathrm{m}$ InAsGaAs quantum-dot lasers with very low continuous-wave room- 
temperature threshold currents. IEEE Photonic Technol. Lett. 17, 1139-1141 (2005)

4. Li, S.G., Gong, Q., Cao, C.F., Wang, X.Z., Yan, J.Y., Wang, Y., Wang, H.L.: A review of external cavity-coupled quantum dot lasers. Springer J. Opt. Quantum Electron. 46, 623-640 (2014)

5. Fathpour, S., Mi, Z., Bhattacharaya, P., Kovsh, A.R., Mikhrin, S.S., Krestnikov, I.L., Kozhukhov, A.V., Ledentsov, N.N.: The role of Auger recombination in the temperature-dependent output characteristics $\left(T_{0}=\infty\right)$ of p-doped $1.3 \mu \mathrm{m}$ quantum dot lasers. Appl. Phys. Lett. 85, 5164-5166 (2004)

6. Jin, C.Y., Badcock, T.J., Liu, H.Y., Groom, K.M., Royce, R.J., Mowbray, D.J., Hopkinson, M.: Observation and modeling of a room-temperature negative characteristic temperature $1.3 \mu \mathrm{m}$ p-type modulation doped quantum dot laser. IEEE J. Quantum Electron. 42, 1259-1265 (2006)

7. Arsenijevic, D., Liu, C., Payusov, A., Stubenrauch, M., Bimberg, D.: Temperature-dependence characteristics of single-mode InAs submonolayer quantum-dot laser. IEEE J. Photonic Technol. Lett. 23, 906-908 (2012)

8. Jin, C.Y., Ohta, S., Hopkinson, M., Kojima, O., Kita, T., Wada, O.: Temperature-dependent carrier tunneling for self-assembled InAs/GaAs quantum dots with a GaAsN quantum well injector. Appl. Phys. Lett. 96, 151104 (2010)

9. Sugawara, M., Mukai, K., Nakata, Y., Otsubo, K., Ishikawa, H.: Performance and physics of quantum-dot lasers with selfassembled columnar-shaped and $1.3 \mu \mathrm{m}$ emitting InGaAs quantum dots. IEEE J. Sel. Topics Quantum Electron. 6, 462-473 (2000)

10. Sugawara, M., Mukai, K., Nakata, Y., Ishikava, H., Sakamoto, A.: Effect of homogeneous broadening of optical gain on lasing spectra in $\mathrm{In}_{x} \mathrm{Ga}_{1-x} \mathrm{As} / \mathrm{GaAS}$ quantum dot lasers. Phys. Rev. B Condens. Matter 61, 7595-7603 (2000)

11. Aryanto, D., Othaman, Z., Ismail, A.K.: The impact of $\mathrm{AsH}_{3}$ overflow time and indium composition on the formation of selfassembled $\mathrm{In}_{x} \mathrm{Ga}_{1-x}$ As quantum dots studied by atomic force microscopy. J. Theor. Appl. Phys. 7, 1-6 (2013)

12. Tan, C.L., Wang, Y., Djie, H.S., Ooi, B.S.: Role of gain broadening in the broadband semiconductor quantum-dot laser. Appl. Phys. Lett. 91, 061117 (2007)

13. Tan, C.L., Wang, Y., Djie, H.S., Ooi, B.S.: The role of optical gain broadening in the ultrabroadband $\mathrm{InGaAs} / \mathrm{GaAs}$ quantumdot laser. Sci. Direct Comput. Mater. Sci. 44, 167-173 (2008)

14. Tan, C.L., Wang, Y., Djie, H.S., Ooi, B.S.: The spectral analysis and threshold limits of quasi-supercontinuum self-assembled quantum dot interband lasers. IEEE J. Quantum Electron. 45, 1168-1176 (2009)

15. Veselinov, K., Grillot, F., Miska, P., Homeyer, E., Caroff, P., Platz, C., Even, J., Marie, X., Dehaese, O., Loualiche, S., Ramdane, A.: Carrier dynamics and saturation effect in (113)B InAs/ InP quantum dot laser. Springer J Opt. Quantum Electron. 38, 369-379 (2006)

16. Grillot, F., Veselinov, K., Gioannini, M., Montrosset, I., Even, J., Piron, R., Homeyer, E., Loualiche, S.: Spectral analysis of $1.55 \mu \mathrm{m}$ InAs-InP (113)B quantum-dot lasers based on a multipopulation rate equation model. IEEE J. Quantum Electron. 45, 872-878 (2009)

17. Gionnini, M., Sevega, A., Montrosset, I.: Simulation of differential gain and linewidth enhancement factor of quantum dot semiconductor lasers. Springer J. Opt. Quantum Electron. 38, 381-394 (2006)
18. Tan, C.L., Wang, Y., Djie, H.S., Ooi, B.S.: Simulation of characteristics of broadband quantum dot lasers. Springer, journal of Optical and Quantum Electronics 40, 391-395 (2008)

19. Bhattacharaya, P., Ghosh, S., Pradhan, S., Singh, J., Wei, Z., Urayama, J., Kim, K., Norris, T.B.: Carrier dynamics and highspeed modulation properties of tunnel injection InGaAs-GaAs quantum dot lasers. IEEE J. Quantum Electron. 39, 952-962 (2003)

20. Su, H., Zhang, L., Gray, A.L., Wang, R., Varangis, P.M., Lester, L.F.: Gain compression coefficient and above-threshold linewidth enhancement factor in InAs/GaAs quantum dot DFB lasers. Proc. SPIE 5722, 72-79 (2005)

21. Fiore, A., Markus, A.: Differential gain and gain compression in quantum-dot lasers. IEEE J. Quantum Electron. 43, 287-294 (2007)

22. Grillot, F., Dagens, B., Provost, J., Su, H., Lester, L.: Gain compression and above-threshold linewidth enhancement factor in $1.3 \mu \mathrm{m}$ InAs-GaAs quantum dot lasers. IEEE J. Quantum Electron. 44, 946-951 (2008)

23. Shafieenezhad, A., Rajaei, E., Yazdan, S.: Impact of gain compression on modulation response and dynamic properties of three state lasing InGaAs/GaAs quantum dot lasers. Opt. Spectrosc. 120, 639-645 (2016)

24. Sugawara, M., Mukai, K., Shoji, H.: Effect of photon bottleneck on quantum-dot laser performance. Appl. Phys. Lett. 71, 2791-2793 (1997)

25. Veselinov, K., Grillot, F., Gioannini, M., Montrosset, I., Homeyer, E., Piron, R., Even, J., Bekiarski, A., Loualiche, S.: Lasing spectra of $1.55 \mu \mathrm{m}$ InAs/InP quantum dot lasers: theoretical analysis and comparing with the experiments. Springer J Opt Quantum Electron 40, 227-22237 (2008)

26. Sugawara, M.: Self-Assembled InGaAs/GaAs Quantum Dots. Academic, New York (1999)

27. Abbaspour, H., Yavari, M.H., Ahmadi, V.: Analysis of QD VCSEL dynamic characteristics considering homogeneous and inhomogeneous broadening. IEEE J. Sel. Top. Quantum Electron. 17, 1327-1333 (2011)

28. Yavari, M.H., Ahmadi, V.: Effects of carrier relaxation and homogeneous broadening on dynamics and modulation behavior of self-assembled quantum-dot laser. IEEE J. Sel. Top. Quantum Electron. 17, 1153-1157 (2011)

29. Mao, M.H., Wu, T.Y., Wu, D.C., Chang, F.Y., Lin, H.H.: Relaxation oscillation and damping factor of $1.3 \mu \mathrm{m} \mathrm{In(Ga)As/}$ GaAs quantum-dot lasers. Springer J. Opt. Quantum Electron. 36, 927-933 (2004)

30. Gready, D., Eisenstein, G., Gionnini, M., Montrosset, I., Arsenijevic, D., Schmeckebier, H., Stubenrauch, M., Bimberg, D.: On the relationship between small and large signal modulation capabilities in highly nonlinear quantum dot lasers. Appl. Phys. Lett. 102, 101107-101109 (2013)

31. Wang, C., Grillot, F., Even, J.: Impact of wetting layer and excited state on the modulation response of quantum-dot lasers. IEEE J. Quantum Electron. 45, 1144-1150 (2012)

32. Grillot, F., Wang, C., Naderi, N.A., Evan, J.: Modulation properties of self-injected quantum-dot semiconductor diode lasers. IEEE J. Sel. Topics Quantum Electron. 19, 1900812 (2013)

33. Mahmoud, S.W.Z.: Influence of gain suppression on static and dynamic characteristics of laser diodes under digital modulation. Egypt J. Solids 30, 237-251 (2007) 